\title{
ASSOCIATION OF CARDIAC STRUCTURAL AND FUNCTIONAL ABNORMALITIES WITH GESTATIONAL DIABETES
}

\author{
Henrique Dória de Vasconcellos, MD, MSc*, Antônio Marconi Leandro da Silva, MD, MSc**, \\ Jeová Cordeiro de Moraes Júnior, MD**, Anderson da Costa Armstrong, ${ }^{*}$ MD, MSc, PhD ${ }^{* *}$, \\ Edgar Guimarães Victor, ${ }^{*} M D, P h D^{* * *}$ \\ Corresponding author: Henrique D. Vasconcellos - henriquedoria@globo.com \\ * Universidade Federal do Vale do São Francisco, Petrolina, Brazil. Universidade Federal de Pernambuco, Recife, Brazil \\ ** Universidade Federal do Vale do São Francisco, Petrolina, Brazil \\ *** Universidade Federal de Pernambuco, Recife, Brazil
}

\begin{abstract}
Introduction: Pregnancy leads to changes in the cardiovascular system. Gestational diabetes relates to maternal and fetus later development of chronic cardiovascular diseases. We perform an exploratory analysis to assess the prevalence of cardiac structural and functional abnormalities in pregnant women with gestational diabetes. Methods: We enrolled 20 consecutive pregnant women with gestational diabetes and 30 healthy pregnant women as controls. Participants with age less than 18 years, previous gestational diabetes, hypertension, cardiac diseases, lung diseases, or medications other than vitamins were excluded. A comprehensive echocardiography protocol was performed in all participants, including $2 \mathrm{D}$ views, $M$-mode technique, flow assessment, and tissue Doppler imaging to acquire cardiac structural and functional parameters. A cross-sectional analysis was performed to assess relations of gestational diabetes with echocardiography-derived parameters. Results: Women with gestational diabetes had higher weight compared to normal pregnant participants, but the cardiovascular risk profile was similar in both groups. LV mass index was significantly higher in women with gestational diabetes when compared to normal pregnant controls, but the other echocardiography-derived parameters were similar in both groups. Gestational diabetes had significant predictive power on higher values of LV mass index, consistent after adjustment for anthropometrics, risk factors, and gynecological history. Conclusion: The presence of gestational diabetes has a consistent independent relation with higher values of LV mass index, but not with other measurements of LV systolic and diastolic function. Myocardial hypertrophy appears to be an early marker of gestational diabetes-related cardiac injury.
\end{abstract}

Keywords: Gestational diabetes; Cardiovascular dysfunction; Left ventricular remodeling; Echocardiography. 


\section{INTRODUCTION}

Pregnancy leads to hemodynamic changes in the cardiovascular system. These hemodynamic changes relate to cardiac adaptive remodeling, persisting a long period after the termination of pregnancy. In addition, cardiovascular diseases are a major concern during pregnancy, being related to a high mortality risk. ${ }^{(1)}$

Diabetes is a strong risk factor for atherosclerosis and coronary artery disease. ${ }^{(2)}$ Diabetes also relates to cardiac fibrosis and remodeling, which may be implicated in early subclinical cardiac dysfunction. ${ }^{(3)}$ However, it is unclear how short-term exposition to diabetes affects the heart morphology and function.

The diagnosis of gestational diabetes is restricted to the pregnancy period, but has shown relation to cardiac abnormalities. In fact, pregnancy-related hyperglycemia appears to be associated to maternal weight and elevated blood pressure. (4) Maternal gestational diabetes also relates to susceptibility of the fetus to later develop cardiovascular risk factors. (5) Furthermore, few studies show how gestational diabetes may influence additional cardiac changes beyond the pregnancy-related adaptive response.

To aid understanding the associations of pregnancy-related diabetes and heart disease, we perform an exploratory analysis to assess the prevalence of cardiac remodeling and dysfunction in pregnant women with gestational diabetes.

\section{METHODS}

\section{STUDY POPULATION}

In a tertiary care hospital specialized in women health, we enrolled 20 consecutive pregnant women with gestational diabetes and 30 healthy pregnant women as controls. The diagnosis of gestational diabetes was established following the Brazilian Society of Diabetes and Brazilian Federation of Gynecology and Obstetrics (FEBRASCO) criteria. $^{(6)}$ Exclusion criteria for both groups were: age less than 18 years, previous gestational diabetes, hypertension, coronary disease, valvular heart diseases, rheumatic diseases, lung diseases and medications other than vitamins. Elected participants underwent a physical examination to compute rest blood pressure, height, and weight. Additional information on previous heart disease and gestational age were self-reported. The institution's Ethical Committee approved the study and all patients signed an informed consent.

\section{ECHOCARDIOGRAPHY PROTOCOL}

A single expert performed and read all transthoracic echocardiograms. The images were obtained at rest with the patient in the supine and left lateral position, using a single scanner, Envisor $C^{\circledR}$ (Philips, Andover, MA, USA). Image acquisition and analysis followed recommendations from the American Society of Echocardiography (ASE), ${ }^{(7,8)}$ using standard tomography slices (parasternal longitudinal and transverse, apical, subcostal and suprasternal) and a multifrequency transducer 2 to $4 \mathrm{MHz}$, in harmonic mode.

Left atrium volume (LAV) was measured by biplane area-length method, using apical 2- and 4-chamber views, and then indexed by body surface area (BSA). Aortic root was assessed using M-mode echocardiography. LV mass was assessed using 2D-guided $\mathrm{M}$-mode measurements and were also indexed by BSA. LV ejection fraction was determined by the Teicholz method, using 2D-guided M-mode measurements.

Pulsed Wave Doppler sample volumes (2mm) were located at the ends of the leaflets of the mitral valve and between the inlet and the LV outflow tract. Then, early diastolic influx peak $(E)$ and late diastolic peak (A) were assessed. The isovolumetric relaxation time (IVRT) and the $\mathrm{E}$ wave deceleration time (DT) were also computed, and the E/A ratio was calculated. Mitral valve inflow also was assessed in 4-chamber views by color Doppler M-mode and the propagation velocity (PV) was measured. Tissue Doppler imaging (TDI) was obtained from the septal and the lateral regions of the mitral annulus and then averaged to compute e' values. 


\section{STATISTICAL ANALYSIS}

Continuous variables were described by means and standard deviations (SD) and the categorical variables were reported as proportions. Univariate analysis compared echocardiography-derived parameters in participants with gestational diabetes and without gestational diabetes, using t-test or Wilcoxon rank-sum test. Multivariable analysis was conducted if any significance $(p<0.05)$ found in univariate analysis. Multivariable linear regression models assessed the ability of gestational diabetes (independent variable) to predict significant echocardiography-derived parameters (dependent variable), adjusting for anthropometrics, known cardiovascular risk factors, and gynecological history. For this purpose, we used two models: Model 1 -adjusted for weight, age, and systolic blood pressure; Model 2 - Model $1+$ number of pregnancies, number of deliveries, and gestational age. All cross-sectional analyzes were performed using STATA v. 11 software.

\section{RESULTS}

Women with gestational diabetes had higher weight, compared to normal pregnant women. Age and the cardiovascular risk profile were similar between groups (Table 1).

Table 1 - Description of participant clinical characteristics

\begin{tabular}{lcc}
\hline \multicolumn{1}{c}{ Parameter } & $\begin{array}{c}\text { CASES }(\mathrm{N}=\mathbf{2 0}) \\
(\mathrm{MEAN}+/-\mathrm{SD})\end{array}$ & $\begin{array}{c}\text { Controls }(\mathrm{N}=30) \\
\text { (MEAN+/- SD) }\end{array}$ \\
\hline Age (years) & $29.75+/-4.05$ & $27.7+/-5.91$ \\
Weight* $(\mathrm{Kg})$ & $76.80+/-11.17$ & $70.17+/-10.08$ \\
Height (m) & $161.10+/-4.96$ & $160.83+/-6.87$ \\
Number of pregnancies & $2+/-1.17$ & $1.73+/-0.83$ \\
Gestational age (weeks) & $31.85+/-3.34$ & $32.27+/-3.64$ \\
Heart Rate (beats per minute) & $87.70+/-9.08$ & $84.53+/-10.33$ \\
Systolic blood pressure (mmHg) & $121.50+/-11.82$ & $116.33+/-11.52$ \\
Diastolic blood pressure (mmHg) & $74.25+/-9.07$ & $70.50+/-9.41$ \\
Gestational age (weeks) & $32+/-3.3$ & $32+/-3.6$ \\
\hline
\end{tabular}

$* p$ value $=0.034$. For all other parameters, $p$ value $>0.05$

In the univariate analysis, LV mass index was significantly higher in women with gestational diabetes when compared to normal pregnant controls.
A similar profile for women with gestational diabetes and controls was found for other echocardiographyderived parameters (Table 2). 
Table 2 - Description of echocardiography parameters in participants with gestational diabetes $(n=20)$ and normal pregnant women $(n=30)$

\begin{tabular}{|c|c|c|c|}
\hline PARAMETER & $\begin{array}{l}\text { CASES }(N=20) \\
(\text { MEAN+/-SD) }\end{array}$ & $\begin{array}{c}\text { CONTROLS }(\mathrm{N}=30) \\
(\mathrm{MEAN}+/-\mathrm{SD})\end{array}$ & PARAMETER \\
\hline Aortic root (mm) & $28.4+/-2.9$ & $27.9+/-2.9$ & 0.525 \\
\hline LV mass index $(\mathrm{g} / \mathrm{m} 2)$ & $47.3+/-8.6$ & $41.8+/-8.7$ & O.031 \\
\hline LVEF (\%) & $67.9+/-1.9$ & $68.0+/-3.4$ & 0.89 \\
\hline LA volume index $(\mathrm{mL} / \mathrm{m} 2)$ & $16.8+/-2.3$ & $16.3+/-2.0$ & 0.44 \\
\hline E/A ratio & $1.4+/-0.3$ & $1.5+/-0.3$ & 0.382 \\
\hline DT (ms) & $164.6+/-33.3$ & $164.9+/-33.4$ & 0.977 \\
\hline IVRT (ms) & $88.1+/-13.1$ & 81.0 +/- 11.4 & 0.050 \\
\hline $\mathrm{PV}(\mathrm{cm} / \mathrm{s})$ & $70.4+/-17.3$ & $71.2+/-12.2$ & 0.862 \\
\hline$e^{\prime}$ medial $(\mathrm{cm} / \mathrm{s})$ & $10.2+/-2.9$ & $11.3+/-2.7$ & 0.203 \\
\hline$e^{\prime}$ lateral $(\mathrm{cm} / \mathrm{s})$ & $19.0+/-12.1$ & $16.3+/-4.5$ & 0.263 \\
\hline$E / e^{\prime}$ & $6.68+/-2.3$ & $6.66+/-1.6$ & 0.507 \\
\hline
\end{tabular}

LVEF - left ventricular ejection fraction; LA - left atrial; DT - deceleration time; IVRT - isovolumetric relaxation time; $\mathrm{PV}$ - propagation velocity; In E / E' ratio, e' is the average of medial and lateral e'.

Multivariable linear regression assessed the relations between gestational diabetes and LV mass index (Table 3). Presence of gestational diabetes showed significant predictive power on higher values of LV mass index. These findings were consistent after adjusting for anthropometrics, risk factors, and gynecological history.

Table 3 - Multivariable linear regression analysis $(n=50)$ for the association of gestational diabetes and left ventricular mass index $(\mathrm{g} / \mathrm{m} 2)$

\begin{tabular}{lccccc}
\hline \multirow{2}{*}{ PARAMETER } & \multicolumn{2}{c}{ MODEL 1 } & & \multicolumn{2}{c}{ MODEL 2 } \\
\cline { 2 - 3 } \cline { 5 - 6 } \cline { 5 - 6 } & COEFF & $\mathbf{P}$ & & COEFF & P \\
\hline Gestational diabetes & 6.00 & $\mathbf{0 . 0 3 3}$ & & 6.05 & $\mathbf{0 . 0 3 6}$ \\
Weight (Kg) & 0.04 & 0.743 & & -0.02 & 0.917 \\
Age (years) & -0.16 & 0.531 & & -0.29 & 0.321 \\
SBP (mmHg) & -0.08 & 0.514 & & -0.09 & 0.463 \\
Number of pregnancies & & & & 4.45 & 0.175 \\
Number of deliveries & & & & -4.65 & 0.243 \\
Gestational age (weeks) & & & & -0.03 & 0.934 \\
\hline
\end{tabular}

SBP. Systolic blood pressure. Model 1 - adjusted for weight, age, systolic blood pressure. Model 2 - Model $1+$ number of pregnancies, number of deliveries, and gestational age.

\section{DISCUSSION}

In this study, we investigate a group of women with gestational diabetes compared to a control of normal pregnant women and show their echocardiographic profiles. Gestational diabetes was consistently associated with left ventricular hypertrophy. Other echocardiography-derived cardiac parameters did not show significant relation with the presence of gestational diabetes.

Diabetes mellitus is a group of metabolic diseases characterized by chronic hyperglycemia, often associated with endothelial dysfunction and atherosclerosis. ${ }^{(2)}$ Gestational diabetes is defined 
as carbohydrate intolerance, in varying degrees of intensity, started or first diagnosed during pregnancy and its prevalence is around $7 \%$ of the pregnancies in Brazil. ${ }^{(9,10)}$ Development of gestational diabetes is a predictor for future diagnosis of type II diabetes and metabolic syndrome. ${ }^{(6)}$ However, the effects of gestational diabetes on cardiac structure and function are poorly understood.

Our study did not find a significant associations of gestational diabetes with cardiac dysfunction, although a previous report has shown longterm relations between gestational diabetes and diastolic dysfunction. (11) Diabetes-related cardiac remodeling may be mediated by time of exposition to hyperglycemia. ${ }^{(12)}$ Therefore, a longer follow-up could be needed to find cardiac dysfunction in our study, possibly related to an increase in interstitial cardiac fibrosis. ${ }^{(3)}$

Remarkably, the presence of gestational diabetes was a consistent predictor of left ventricular hypertrophy, as assessed by an increased LV mass index. In fact, echocardiography-derived LV mass and hypertrophy are validated, widely available, and accurate measurements. ${ }^{(13,14)}$ Moreover, LV mass is a powerful predictor of major cardiovascular outcomes; even in early adulthood, when CV risk factors exposition is still incipient. ${ }^{(15,16)}$ Therefore, LV mass index raises as an early marker of cardiac remodeling in patients with gestational diabetes. Myocardial hypertrophy is an important marker of cardiac remodeling and may be presented in the earlier stages of gestational diabetes-related cardiac injury; while diastolic and systolic dysfunction appear later, after the heart loses its functional compensatory mechanisms.

In conclusion, we investigated a group of women with the diagnosis of gestational diabetes and identified a consistent independent relation between the presence of gestational diabetes and higher values of LV mass index. Measurements of LV systolic and diastolic function did not correlate to gestational diabetes in our study. Myocardial hypertrophy appears to be an early marker of gestational diabetes-related cardiac injury.

\section{REFERENCES}

1. Alonso-Gonzalez R, Swan L. Treating cardiac disease in pregnancy. Womens Health. 2014;10(1):79-88; quiz 89-90.

2. Ladeia AM, Sampaio RR, Hita MC, Adan LF. Prognostic value of endothelial dysfunction in type 1 diabetes mellitus. World J Diabetes. 2014;5(5):601-5.

3. Turkbey EB, Backlund JY, Genuth S, Jain A, Miao C, Cleary PA, Lachin JM et al. Myocardial Structure, Function, and Scar in Patients With Type 1 Diabetes Mellitus. Circulation. 2011;124(16):1737-1746.

4. Kaul P, Savu A, Nerenberg KA, Donovan LE, Chik CL, Ryan EA, Johnson JA. Impact of gestational diabetes mellitus and high maternal weight on the development of diabetes, hypertension and cardiovascular disease: a population-level analysis. Diabet Med. 2014.

5. Chavey A, Ah Kioon MD, Bailbe D, Movassat J, Portha B. Maternal diabetes, programming of beta-cell disorders and intergenerational risk of type 2 diabetes. Diabetes Metab. 2014.

6. Bertoluci MC, Pimazoni-Netto A, Pires AC, Pesaro AE, Schaan BD, Caramelli B, Polanczyk $C A$ et al. Diabetes and cardiovascular disease: from evidence to clinical practice - position statement 2014 of Brazilian Diabetes Society. Diabetol Metab Syndr. 2O14;6:58.

7. Lang RM, Bierig M, Devereux RB, Flachskampf FA, Foster E, Pellikka PA, Picard MH et al. Recommendations for chamber quantification: a report from the American Society of Echocardiography's Guidelines and Standards Committee and the Chamber Quantification Writing Group, developed in conjunction with the European Association of Echocardiography, a branch of the European Society of Cardiology. J Am Soc Echocardiogr. 2005;18(12):1440-63.

8. Nagueh SF, Appleton CP, Gillebert TC, Marino PN, Oh JK, Smiseth OA, Waggoner $A D$ et al. Recommendations for the evaluation of left ventricular diastolic function by echocardiography. J Am Soc Echocardiogr. 2009;22(2):107-33.

9. Maraschin Jde F. Classification of diabetes. Adv Exp Med Biol. 2012;771:12-9. 
1O. Schmidt MI, Duncan BB, Reichelt AJ, Branchtein L, Matos MC, Costa e Forti A, Spichler ER et al. Brazilian Gestational Diabetes Study G. Gestational diabetes mellitus diagnosed with a 2-h 75-g oral glucose tolerance test and adverse pregnancy outcomes. Diabetes Care. 2001;24(7):1151-5.

11. Freire CM, Nunes M do C, Barbosa MM, Longo JR, Nogueira AI, Diniz SS, Machado LJ et al. Gestational diabetes: a condition of early diastolic abnormalities in young women. J Am Soc Echocardiogr. 2006;19(10):1251-6.

12. Armstrong $A C$, Liu $K$, Lewis $C E$, Sidney $S$, Colangelo LA, Kishi S, Ambale-Venkatesh B et al. Left atrial dimension and traditional cardiovascular risk factors predict 2O-year clinical cardiovascular events in young healthy adults: the CARDIA study. Eur Heart J Cardiovasc Imaging. 2014;15(8):893-9.

13. Armstrong $A C$, Gjesdal $O$, Almeida A, Nacif $M$, Wu C, Bluemke DA, Brumback $L$ et al. Left ventricular mass and hypertrophy by echocardiography and cardiac magnetic resonance: the multi-ethnic study of atherosclerosis. Echocardiography. 2014;31(1):12-2O.

14. Armstrong AC, Ricketts EP, Cox C, Adler P, Arynchyn A, Liu K, Stengel E et al. Quality Control and Reproducibility in M-Mode, Two-Dimensional, and Speckle Tracking Echocardiography Acquisition and Analysis: The CARDIA Study, Year 25 Examination Experience. Echocardiography. 2014.

15. Armstrong $A C$, , Gjesdal $O$, Wu $C$, Gidding $S$, Bluemke DA, Lima JA. Left ventricular mass assessed by Echocardiography and Cardiac Magnetic Resonance, cardiovascular outcomes, and medical practice. JACC Cardiovasc Imaging 2O12;5(8):837-48.

16. Armstrong AC, Junior Jacobs DR, Gidding SS, Colangelo LA, Gjesdal O, Lewis CE, BibbinsDomingo $\mathrm{K}$ et al. Framingham score and LV mass predict events in young adults: CARDIA study. Int J Cardiol 2O14;172(2):350-5. 